\title{
Considering temporal dimension for NSCLC medical management?
}

\author{
Focan C*, Bourhaba M, Graas MP and Levi F \\ ${ }^{1} \mathrm{CHC}$-Liège. ESMO Integrated Center for Oncology and Palliative Care, Clinique Saint-Joseph, rue de Hesbaye 75 - B-4000 LIEGE, Belgium \\ ${ }^{2}$ Cancer Chronotherapy Unit, Warwick University Medical School, Hill Road ; Coventry CV4 7AL-Warwick - UK
}

Life is structured in space but also in time. Biological rhythms, ie circadian ones, have been documented in all processes involved in the malignant transformation of cells as well as in the cellular proliferation of both healthy and tumor tissues [1].

Recent advances identify critical molecular events that rhythmically control drug metabolism and detoxification, cell cycle, molecular targets, DNA (deoxyribonucleic acid) repair, apoptosis, and angiogenesis [2-5]. The coordination of these processes along the 24-h period is ensured by the circadian timing system (CTS) [1-3]. The CTS coordinates physiology and cellular functions over a $24 \mathrm{~h}$ period. This circadian physiology is generated and controlled by a central pacemaker, the suprachiasmatic nuclei (SCN) in the hypothalamus , through diffusible signals, including transforming growth factor alpha (TGF-alpha), epidermal growth factor (EGF), prokineticin-2, cardiotrophin-like cytokine and neuroanatomic sympathetic and parasympathetic pathways (4).A dozen specific clock genes constitute the core of the molecular clock in mammals. In particular, the CLOCKBMAL1 (circadian locomotor output cycles kaput-brain and muscle ARNT like protein-1) or NPAS2-BMAL1 protein dimers play a key role in the molecular clock through the activation of transcriptional clock genes Period's (Per's), Cryptochrome (Cry's), and Reverb's .In mammals, the core clock genes, Per1 and Per2, are key regulators of circadian rhythms both in central clock, in hypothalamus and in peripheral tissues [1-4]. Recent findings revealed molecular links between Per genes and cellular components that control fundamental cellular processes such as cell division and DNA damage.

Disregulation of circadian rhythms might influence the susceptibility to cancer development (breast, colorectal, lung, prostate...) and provide further support for the emerging role of circadian genes in tumor suppression [5]. Precisely, silencing of tumor suppressor genes, such as the Per's - clock core gene, resulting from epigenetic alterations may occur early in lung cancer tumorigenesis. Alterations of sleep quality and disruption of melatonin circadian rhythms (peak at night after dim-lighting) may enhance the risk of various cancers including lung ones [6].

Various mechanisms responsible for the deregulation of cell cycle and enhanced susceptibility to oncogenesis through activation of cell proliferation and cancer promotion have been identified. For example, in NSCLC, over-expression of cyclin D1 and mutation of p16 leading to a shortened and accelerated G1-phase and permanent phosphorylation and inactivation of $\mathrm{pRb}$ have been observed. Per1 and Per2 exert their tumor suppressor functions in a circadian time-dependent manner [7]. Their overexpression inhibits neoplastic growth both in vitro and in vivo and increases apoptosis while their down regulation enhanced tumor development and growth. Monitoring of circadian rhythm in VEGF production may be useful for choosing the most appropriate time of day (i.e. when VEGF production is increased) for administrating antiangiogenic agents. WNT10A (wingless gene 10A) could stimulate growth of both microvascular endothelial cells and fibroblasts in tumors along with marked increases in angio/stromagenesis [7]. Circadian disruption may induce progression of malignant tumors via a WNT $10 \mathrm{~A}$ signaling pathway allowing microvascular endothelial cells and fibroblasts in models involving tumor cells similar to that encountered in human NSCLC [8].

Parameters and dissociation constant of EGF-R (epithelial growth factor receptor) exhibit circadian variations [9]. EGF-R by itself was found to phase shift the prominent circadian rhythm of DNA synthesis, i.e. in the esophagus. Also gene expression response to EGF-R is circadian-time dependent. EGF-R and its ligand transforming growth factor-alpha (TGF- $\alpha$ ) are highly expressed in suprachiasmatic nucleus where they exert a circadian-time-dependent neuromodulatory function [4]. The core clock proteins BMAL1, CLOCK, and REV$\mathrm{ERB} \alpha$, controls fundamental aspects of the immune response $[10,11]$. As a matter of fact, Bmall deficiency in macrophages increases PKM2 (pyruvate kinase M2) expression and lactate production, which is required for expression of the immune checkpoint protein PDL1(programmed cell death-ligand1) [10,11]. Also Clock as a member of histone acetyltransferases, controls acetylation of histone 4 required for repair of DNA double strand breaks thanks to several repair genes such as ERCC1(excision repair cross-complementing group 1) or AP1( activator protein 1) [12]; its expression is associated with cisplatin resistance $[12,13]$.

Circadian variation in pharmaco-kinetics (PK) has been evidenced in rodents and later in humans for all drugs routinely used in LC patients, i.e. pyrimidine derivatives, anthracyclines, vincaalkaloïds (vinorelbine), topo-isomerase inhibitors, taxanes, platinum derivatives, gemcitabine and other antimetabolites $[2,3,14,15]$. Also chronotolerance and even chronoefficacy have been observed in rodent studies long time ago for any chemotherapy agents active in NSCLC $[2,3,14,15]$. Diurnal varying pharmacokinetics of erlotinib (a largely used TKI - tyrosine kinase inhibitor for treating human NSCLC)

${ }^{\star}$ Correspondence to: Focan C, CHC-Liège. ESMO Integrated Center for Oncology and Palliative Care, Clinique Saint-Joseph, rue de Hesbaye 75 B-4000 LIEGE, Belgium, Tel: 0032-4-224 89 90; Fax: 0032-4-224089 91; E-mail: christian.focan@chc.be

Received: September 27, 2019; Accepted: October 30, 2019; Published: November 01, 2019 
have also been reported ie in Lewis Lung and in NSCL-xenografts tumor-bearing mice $[16,17]$. Chronotolerance was also reported in 3 different randomized studies in chemotherapy naïve advanced NSCLC patients $(18,19)$. An overall excellent therapeutic index was observed with an infusional chemotherapy for 5 days, with sinusoidal varying administration od 5-fluororacil and carboplatin with the best schedule (peak of 5FU at $4 \mathrm{am}$; peak of carboplatin at $4 \mathrm{pm}$ ) $[18,19]$.

Altered circadian rhythmicity of cortisol, TRH, TSH and GH serum level and all lymphocyte subsets (except CD4) related to the advancing stage of disease as well as a melatonin/cortisol ratio was evidenced in LC patients [20,21]. Circadian time structure like that of control normal subjects seems to be an independent prognostic factor, in advanced breast or colon and also in lung cancer patients $[22,23]$. Circadian rest-activity rhythms as gauged by actometry have shown that circadian destructors measurements were correlated with Quality of Life (QOL), fatigue, altered moods and severe disturbances of daily sleep-activity cycles $[24,25]$. As a matter of fact, in NSCLC, only a few studies dealt with considerations on temporal dimension for drug-delivery: only one limited study has observed better outcome of patients with a circadian sequential schedule; 3 others showed differences in drug dose intensities and /or toxicity but with no evident clinical benefit $[18,19]$. Lissoni and al observed improvement of tumor outcome (response rate; one year survival,...) and QOL by associating to standard cisplatin-based chemotherapy, melatonin and sometimes interleukin-2 [26] . The same observation was provided by Hrushesky's group in a randomized blinded trial on advanced NSCLC patients receiving chemotherapy with etoposide and cisplatin together with melatonin or placebo: those patients receiving melatonin during the evening enjoyed higher response rates (29 vs $8-11 \%$ ) and longer survival in multivariate analysis (personal communication). According to 2 recent meta-analyses, the administration of melatonin reduces the relative risk of death at one year by an average of $37 \%$, doubles the frequency of complete response and reduces the prevalence and/or severity of chemotherapy induced nausea/vomiting, hypotension and hematological toxicity $[27,28]$. Thus some authors considere melatonin as a probable effective treatment for human NSCLC $[27,28]$. In fact, outside its circadian rhythms re-synchronizing properties, melatonin may prevents tumor metastasis via inducing apoptosis processes and restraining the autonomous cell proliferation. Moreover, it inhibits the progression of tumors due to its oncostatic, pro-oxidant and antiinflammatory effects $[27,28]$.

During the last decade, the management of NSCLC has evolved $[29,30]$. Platinum-based chemotherapy remains the standard frontline in treatment of advanced unresectable NSCLC in which cisplatin or carboplatin are combined with another chemotherapeutic agent such as taxanes, pemetrexed or gemcitabine. However results in terms of tumour outcome remained stable over time! [31]. Thus, progresses confirmed by positive phase III trials, came from targeted and immunotherapeutic biological approaches [29,30]. Targeted therapies against EGFR mutations and anaplastic lymphoma kinase (ALK) gene re-arrangement have improved the survival in the small proportion of patients whose tumors were expressing these molecular abnormalities [29]. Also recent development and success of immune check-point inhibition programmed cell death 1 (PD-1), programmed death-ligand 1 (PD-L1) and cytotoxic T-lymphocyte antigen 4 (CTLA-4) have opened new pavements for fruitfull research [30,31]. Unfortunately to the best of our knowledge, despite precise theoretical observations depicted above related to circadian expression, both on targets of TKI-inhibitors, EGF-R blockers, anti-angiogenic agents or
/ on immune active-agents ( lymphoid system; PD1; PDL1), by now no study has been launched taking into account any chronobiological considerations!! However these knowledges would bring hope to still improve overall tumor outcome by optimizing 'classical' therapeutic index of medical therapies but also circadian host rhythmicity by acting on the central clock (ie by TKI administration) and/or molecular machinery (receptors; various enzymatic pathways; DNA metabolism and repairing; immunology pathways.......).

The potential role of melatonin as resynchronizing agent and as a potentially active antitumour agent warrants also further evaluations.

\section{Source}

DOI: http://dx.doi.org/10.5772/intechopen.85710

\section{References}

1. Hastings MH, Maywood ES, Brancaccio M (2019) The Mammalian Circadian Timing System and the Suprachiasmatic Nucleus as Its Pacemaker. Biology 8: E13.

2. Innominato PF, Roche VP, Palesh OG (2014) The circadian timing system in clinical oncology. Ann Med 46: 191-207.

3. Ballesta A, Innominato PF, Dallmann R, Rand DA, Levi FA (2017) Systems Chronotherapeutics. Pharmacol Rev 69:161-199.

4. Vadigepalli R, Hao H, Miller GM, Liu H, Schwaber HS (2008) Epidermal growth factor receptor-induced circadian time-dependent gene relation in suprachiasmatic nucleus. Neuroreport 18: 1437-1441.

5. Yang X, Wood PA, Ansell C, Hrushesky WJ (2009) Circadian time-dependent tumor suppressor function of period genes. Integr Cancer Ther 8: 309-316.

6. Luojus MK, Lehto SM, Tolmunen T, Erkkilä AT, Kauhanen J (2014) Sleep duration and incidence of lung cancer in ageing men. BMC Public Health 14: 295. [Crossref]

7. Zhang HY, Yang W, Lu JB (2017) Knockdown of GluA2 induces apoptosis in nonsmall-cell lung cancer A549 cells through the p53 signaling pathway. Oncol Lett 1: $1005-1010$

8. Yasuniwa Y, Izumi H, Wang KY (2010) Circadian disruption accelerates tumor growth and antistromagenesis through a Wnt signaling pathway. PLoS One 5: e15330.

9. Scheving LA, Tsai TH, Cornett LE, Feuers RJ, Scheving LE (1989) Circadian variation of epidermal growth factor receptor in mouse liver. Anat Rec 224: 459-65.

10. Deng W, Zhu S, Zeng L (2018) The Circadian Clock Controls Immune Checkpoin Pathway in Sepsis. Cell Rep 24: 366-378.

11. Curtis AM, Bellet MM, Sassone-Corsi P, O’Neill LA (2014) Circadian clock proteins and immunity. Immunity 40: 178-186.

12. Focan C (1995) Circadian rhythms and cancer chemotherapy. Pharmacol Ther 67 1-52. [Crossref]

13. Levi F, Focan C, Karaboue A (2007) Implications of circadian clocks for the rhythmic delivery of cancer therapeutics. Adv Drug Deliv Rev 59: 1015-35.

14. Gery S, Koeffler HP (2007) The role of circadian regulation in cancer. Cold Spring Harb Symp Quant Biol 72: 459-464. [Crossref]

15. Miyamoto N, Izumi H, Noguchi T (2008) Tip60 is regulated by circadian transcription factor clock and is involved in cisplatin resistance. J Biol Chemis 283: 18218-18226.

16. Lin P, An F, Xu X, Zhao L, Liu L, et al. (2015) Chronopharmacodynamics and mechanisms of antitumor effect induced by erlotinib in xeno-graft-bearing mice. Biochem Biophys Res Commun 460: 362-367.

17. Liu J, Wang CY, Ji SG, Xu X, Wang PP, et al. (2016) Chronopharmacokinetics of Erlotinib and Circadian Rhythms of Related Metabolic Enzymes in Lewis TumorBearing Mice. Eur J Drug Metab Pharmacokinet 41: 627-635. [Crossref]

18. Focan C (2002) Chronobiological concepts underlying the chronotherapy of human lung cancer. Chronobiol Int 19: 253-273.

19. Focan C, Davin AC, Bourhaba M, Graas MP (2019) Integration of Chronobiological Concepts for NSCLC Management. Intechopen. 
20. Mazzoccoli G, Pazienza V, Peipoli A (2012) Alteration of hypothalamic-pituitarythyroid axis function in non-small cell lung cancer patients. Integr Cancer Ther 11: 327-36.

21. Mazzoccoli G, Vendemiale G, De Cata A, Carughi S, Tarquini R (2010) Altered time structure of neuro-endocrine-immune system function in lung cancer patients. $B M C$ Cancer 10: 314 .

22. Sephton SE1, Sapolsky RM, Kraemer HC, Spiegel D (2000) Diurnal cortisol rhythm as a predictor of breast cancer survival. J Natl Cancer Inst 92: 994-1000. [Crossref]

23. Kim KS, Kim YC, Oh IJ (2012) Association of worse prognosis with an aberrant diurnal cortisol rhythm in patients with advanced lung cancer. Chronobiol Int 29: 1109-1120.

24. Focan C, Levi F, Innominato P (2007) Circadian behaviour of host versus tumour outcome. A review. Pathol Biol 55: 178-180

25. Innominato PF, Focan C, Gorlia T (2009) Circadian rhythm in rest and activity: a biological correlate of quality of life and a predictor of survival in patients with metastatic colorectal cancer. Cancer Res 69: 4700-4707.
26. Lissoni P, Messina G, Balestra A (2008) Efficacy of cancer chemotherapy in relation to synchronization of cortisol rhythm, immune status and psychospiritual profile in metastatic non small cell lung cancer. In Vivo 22: 257-262.

27. Ma Z, Yang Y, Fan C, Han J (2016) Melatonin as a potential anticarcinogen for nonsmall-cell lung cancer. Oncotarget 7: 46768-46784.

28. Wang Y, Wang P, Zheng X, Du X. Therapeutic strategies of melatonin in cancer patients: a systematic review and meta-analysis. Onco Targets Ther 11: 7895-7908.

29. Srivastava R, Lebowicz Y, Jamil MO (2018) Targeted agents in the management of small cell lung cancer - present and future. Drugs Today (Barc) 54: 479-488. [Crossref]

30. Bianco A, Malapelle U, Rocco D, Perrotta F, Mazzarella G (2018) Targeting immune checkpoints in non small cell lung cancer. Curr Opin Pharmacol 40: 46-50. [Crossref]

31. Ramalingam SS, Owonikoko TK, Khuri FR (2011) Lung cancer: new biological insights and recent therapeutic advances. CA Cancer J Clin 61: 91-112.

Copyright: $\odot 2019$ Focan C. This is an open-access article distributed under the terms of the Creative Commons Attribution License, which permits unrestricted use, distribution, and reproduction in any medium, provided the original author and source are credited. 